\title{
Effects of material and process parameters on in-situ consolidation
}

\author{
Angel Leon ${ }^{1} \cdot$ Clara Argerich $^{2} \cdot$ Anais Barasinski $^{2} \cdot$ Eric Soccard $^{3} \cdot$ Francisco Chinesta $^{4}$ (i)
}

\begin{abstract}
Automated tape placement - ATP - is a recent manufacturing technology for composite materials. Therefore, a correct modeling of the multi-physical process is critical in order to make possible in-situ consolidation. In this work, we propose an accurate modelling framework and an efficient simulation procedure of physics occurring during the process with the aim of studying the influence of material and process parameters into the material consolidation evolution. For that purpose, an accurate description of the prepreg surface becomes compulsory, justifying the use of a multi-resolution description of it based on the use of wavelets.
\end{abstract}

Keywords Consolidation · Pre-preg $\cdot$ Squeeze flow $\cdot$ PGD $\cdot$ Wavelet surface representation

\section{Introduction}

Automated tape placement is a promising composite forming process based on the fusion bonding of a prepreg tape on a substrate. By heating the interface between the incoming tape and the substrate, and applying pressure, a laminate part, out-of-autoclave, can be obtained in one-shot, making the process attractive for industry.

In order to reach in-situ consolidation, the material has to undergo several steps: heating, consolidation and cooling,

Francisco Chinesta

Francisco.Chinesta@ec-nantes.fr

Angel Leon

aeo@esi-group.com

Clara Argerich

clara.argerich-martin@ec-nantes.fr

Anais Barasinski

anais.barasinski@ec-nantes.fr

Eric Soccard

eric.soccard@airbus.com

1 ESI GROUP, Bristol Composites Institute (ACCIS), Queen's Building, University Walk, Bristol BS8 1TR, UK

2 GeM Institute, Ecole Centrale Nantes, 1 rue de la Noe, F-44300 Nantes, France

3 Airbus SAS, Allee du Chauffault, F-44340 Bouguenais, France

4 PIMM, ENSAM ParisTech, ESI GROUP Chair on Advanced Modeling and Simulation of Manufacturing Processes, 151 Boulevard de l'Hôpital, F-75013 Paris, France as sketched in Fig. 1. During these three stages, different physical phenomena occur, all them taking place at the tapesubstrate interface and are governed by temperature and pressure evolutions [1].

Many studies in the past considered the deformation of rough surfaces squeezed under the action of an external applied pressure, using different representations of rough surfaces $[8,12]$. The characterization of random surfaces and the effect of roughness on physics defined at the surface level is a recurrent issue widely addressed in many works $[13,14]$. Fractals have been largely considered for describing these random surfaces exhibiting self-affinity through many space scales [15-18].

A critical aspect concerns the consolidation step, when the bonding occurs. This aspect widely treated in several works [12, 20-22] considers surface descriptors based on the use of rectangular elements (emulating the asperities) or fractals. However, analyses seem to reveal that those descriptions are not discriminant enough, that is, the same descriptors could represent surface exhibiting very different evolutions of the degree of intimate contact - DIC -. Thus, it seems important to differentiate between geometrical and physical descriptors, the last being goal oriented.

In our previous works, we considered a surface representation based on a fractal Cantor which parameters are extracted from a prepreg experimental measurement. Then it was heated and compressed by a flat plate, allowing the evaluation of the DIC evolution [9-11]. From our analysis we concluded that even if the fractal seems to be providing an appropriate geometrical description, that makes possible 


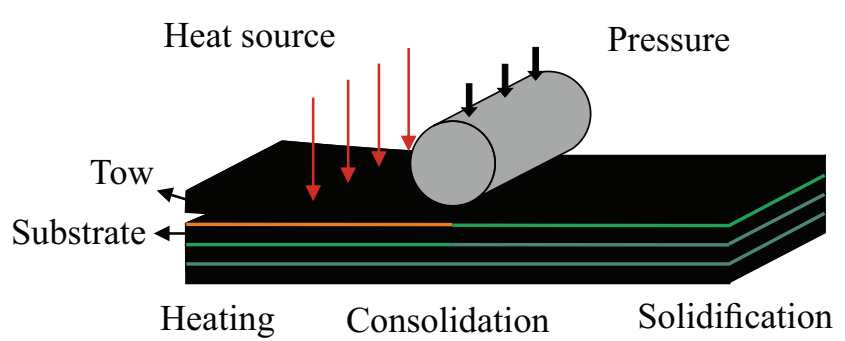

Fig. 1 Sketch of the ATP process

a multi-scale physical analysis when employing adequate numerical techniques, such a description seems limited, and fails in discriminating equivalent surfaces (from the geometrical point of view) that exhibit very different DIC evolutions. Thus, the proposal of goal-oriented descriptors seems compulsory but nowadays almost unachieved.

A possible gateway, in absence of adequate descriptors, consists in making analysis on the real surface geometry. The last is available as soon as it is properly measured by using for example a profilometer. Then, a multi-scale geometrical surface representation could be achieved by using wavelets up to the required level of detail. As soon as the real surface is accurately described from a geometrical view point, thermal and flow analysis can be carried-out by using high-fidelity numerical simulations, based on the use of model order reduction techniques, and more concretely the Proper Generalized Decomposition - PGD - revisited later.

\section{Methods}

In this section we present the two main techniques that will be considered for modelling and simulating the process, the multi-scale representation of the surface and the highresolution thermal analysis.

The multiscale representation of the surface allows considering the last composed of a series of rectangles where the squeeze flow problem will be solved later for simulating consolidation.

\section{Multi-scale representation of pre-impregnated tape surfaces}

The main advantage of representing the real surface using Haar wavelets is that it allows a representation of the real surface as a sequence of rectangles of different height and width. To find more information about wavelet-based multiresolution analysis the reader can refer to [7]. Figure 2 depicts the wavelets representation of a simple profile, where different levels of representation can be observed.

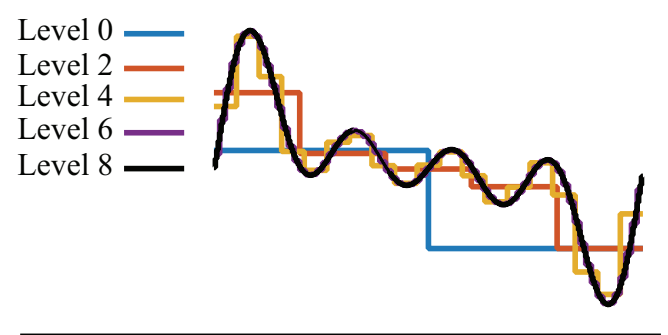

Fig. 2 Simple surface representation using Haar wavelets

When using multi-resolution analysis, the representation levels are embedded, that is, the solution at a certain level contains the ones at the lower levels and is contained in the greater levels representations. The higher is the level, the lower is the width of Haar rectangles representation, allowing for better resolutions. Thus the real surface profile can be represented with the required accuracy by choosing an adequate level of representation. In multi-resolution analysis each level can be written from the previous one by adding its orthogonal complement. When this orthogonal complement becomes small enough, one can consider that the function is adequately represented, as illustrated in Fig. 3 when reaching the 9th-level (the one that allows for an almost perfect representation of the surface when measuring the norm of the difference between the representation and the surface). Such kind of representation, composed of rectangular elements, is very favorable in a double sense: (i) it facilitates a space separated representation within the PGD framework as described in the next section; and (ii) it allows addressing the squeeze flow within the lubrication approximation that instead of solving a $3 \mathrm{D}$ problem in a $3 \mathrm{D}$ domain, solves a $1 \mathrm{D}$ problem in a $2 \mathrm{D}$ domain, described in "High-resolution thermal analysis".

\section{High-resolution thermal analysis}

When considering, as just discussed, the surface represented by a sequence of rectangular elements, high resolution thermal simulations can be performed within the Proper

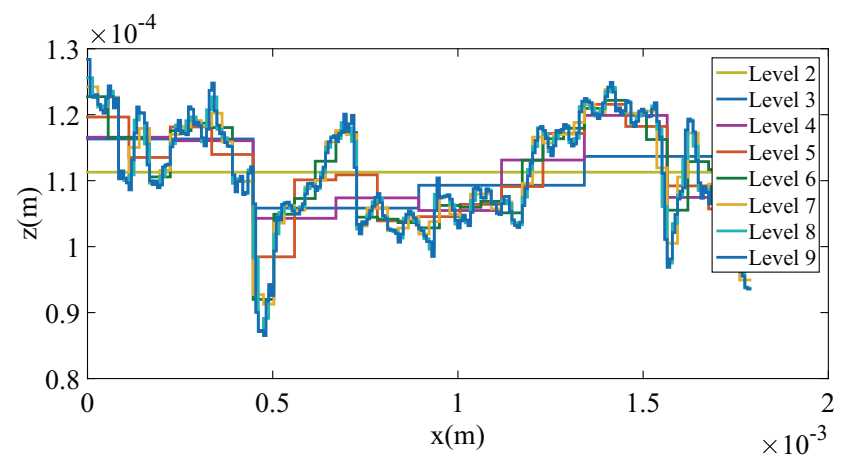

Fig. 3 Real surface representation using the Haar wavelet 


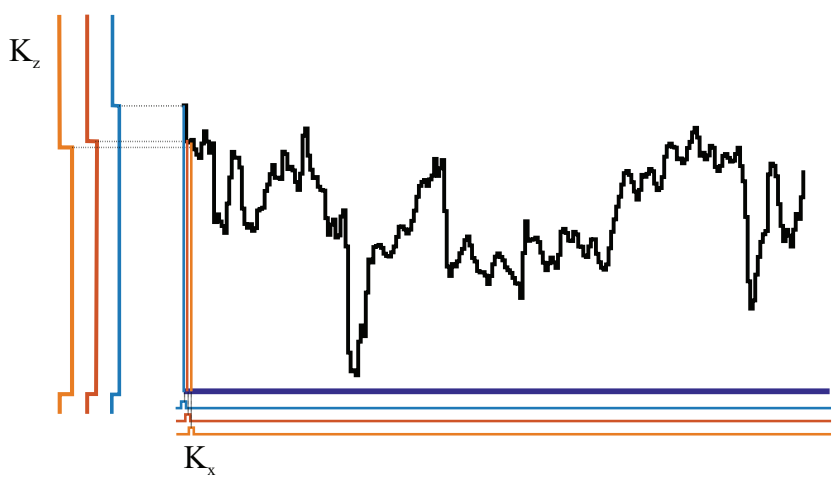

Fig. 4 Separated representation of the thermal conductivity

Generalized Decomposition - PGD - framework by transforming the $2 \mathrm{D}$ heat conduction problem (defined in the layered stacking composed of the already consolidated tapes and the one under consideration) into a sequence of 1D heat transfer problems along directions $x$ and $z$ (see Fig. 5) when assuming a separated representation of both the temperature field and the material thermal properties. For more details about the method, the reader can refers to [3-5].

The reduction of a $2 \mathrm{D}$ problem into a sequence of 1D is compulsory if fine details related to the multi-scale surface representation are retained in the simulation for describing very accurately the evolution of the degree of intimate contact and molecular diffusion at the interface where contact applies.

Within the PGD framework the temperature field $T(x, z)$ is searched in the separated form:

$T(x, z) \approx \sum_{i=1}^{M} X_{i}(x) \cdot Z_{i}(z)$

and the thermal properties, in the present case the thermal conductivity and specific heat capacity, are also expressed using a separated representation, as illustrated in Fig. 4.

$\mathbf{K}(x, z)=\left(1-\sum_{j=1}^{n_{r}} K_{x}^{j}(x) \cdot K_{z}^{j}(z)\right) \mathbf{K}_{c}+\left(\sum_{j=1}^{n_{r}} K_{x}^{j}(x) \cdot K_{z}^{j}(z)\right) \mathbf{K}_{a}$

where $\mathbf{K}_{a}$ and $\mathbf{K}_{c}$ are respectively the thermal air and composite conductivities (in transverse directions to the fibre arrangement), the former assumed isotropic. $K_{x}^{j}$ and $K_{z}^{j}$ are the characteristic functions related to the rectangles occupied by the air in the surface representation (see Fig. 4). In the previous expression, $n_{r}$ is the number of functions required for expressing the void volume as the union of disjoint rectangles. A similar separated representation is carried out for the specific heat capacity.

The transient thermal problem is defined in a rectangular $2 \mathrm{D}$ domain $\Omega$ illustrated in Fig. 4. On the lateral boundaries thermal exchanges with the surrounding air assumed at ambient temperature are considered. On the bottom surface the heat flux from the part to the tool is expressed from a different exchange coefficient and finally on the top boundary the exchange applies first with the roller and when it moves away, with the surrounding air. At the interface level, a heat flux is applied, coming from the laser and acting at the substrate / incoming tape interface. Moreover, thermal conduction between the different plies constituting the substrate is affected from a contact thermal resistance as discussed in our former works $[1,2,6]$.

The heat problem consists in calculating the temperature field $T(x, z)$ verifying:

$C p \cdot \rho \int_{\Omega} T^{*} \frac{\partial T}{\partial t} d \mathbf{x}-\int_{\Omega} T^{*} \nabla(\mathbf{K}(x, z) \nabla T) d \mathbf{x}=0, \quad \forall T^{*}(x, z)$

By integrating by parts the second integral in Eq. 3, natural boundary conditions (heat fluxes) on the domain boundary and interfaces come naturally into the problem. The numerical treatment of thermal contact resistances was deeply addressed in [2].

Because the thermal model is coupled with the squeeze flow occurring when roller compresses the incoming tape, the interface evolves and then the separated representations of the thermal properties must be updated. In summary, the solution of the high-fidelity two-dimensional heat conduction problem can be written as a sequence of the corresponding one-dimensional problems thanks to the separated representation of both the temperature field and the thermal properties representations. As rectangles can be easily separated by considering the product of their respective characteristic functions, a surface representation from Haar wavelets seems an appealing choice from the computational efficiency viewpoint. The use of about one million of nodes in the discretization of the one-dimensional problems defined in $\Omega_{x}$ and $\Omega_{z}$ allows calculating a solution equivalent to the use of $10^{12}$ nodes for discretizing the associated two-dimensional problem in $\Omega=\Omega_{x} \times \Omega_{z}$, and even if the solution of a problem involving $10^{12}$ is unpracticable in most cases, the solution of some one-dimensional problems involving each $10^{6}$ degrees of freedom can be performed in few seconds on a standard laptop.

The importance of fine enough thermal solutions is double. From one side the temperature determines the resin viscosity of major importance when simulating the squeeze flow at the interface level, the last determines the evolution of the intimate contact and then the thermal flux across it. Thus the thermal field affects the resin rheology and the resin flow at the interface level, and the last determines the heat flux across the interface and then the thermal field evolution. Similar analyses were carried out in our former works [9-11] where thermal and flow problems at the interface level were coupled while adopting a Cantor fractal representation of the surfaces. The present work represents a step forward by considering all the multi-physics modelling 


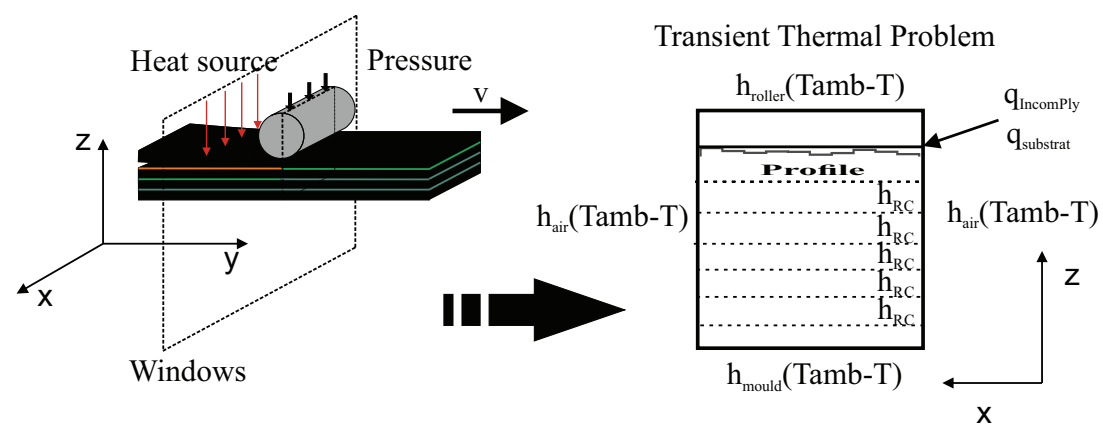

and simulation operating in the real configuration and not in an idealized representation of it.

\section{Surface evolution during the in-situ consolidation}

In this section a model dealing with the evolution of the surface of the pre-impregnated tape during the in-situ consolidation is proposed. It consists in simulating the squeeze flow while assuring the lubrication hypothesis valid.

\section{Squeeze flow of a single rectangular element}

As commented before, the surface is expressed using Haar wavelets, leading to a multi-scale representation that approximates the surface using rectangular elements. This representation is well adapted to the lubrication hypothesis. In the case of a prepreg surface, the height is very low with respect to the width of the domain, at least one order of magnitude as depicted in Fig. 6, validating the lubrication hypothesis discussed later. In what follows, we first revisit the squeeze flow of a rectangular element, and then we consider the assemblage of such elements, assumed representing up-to a certain level of accuracy the real surface. The resulting simulation strategy corresponds to the one considered in [11] for squeezing surfaces described from a Cantor fractal, here extended to a surface wavelet description.

\section{Newtonian fluid}

We consider at time $t$ the rectangular element representing an asperity $\Omega_{t}=\left[-L_{t} / 2, L_{t} / 2\right] \times\left[-H_{t} / 2, H_{t} / 2\right]$ occupied by a Newtonian fluid characterized by a viscosity $\eta$, with, as discussed above, $L_{t} \gg H_{t}$. This element results from the compression of the initial rectangular element $\Omega_{0}=$ $\left[-L_{0} / 2, L_{0} / 2\right] \times\left[-H_{0} / 2, H_{0} / 2\right]$, with $H_{t} L_{t}=H_{0} L_{0}$ ensuring the fluid incompressibility. We assume that on the upper boundary $z=H_{t} / 2$ a normal force applies $\mathbf{F}=$ $-\mathcal{F} \mathbf{e}_{z}$, with $\mathbf{e}_{z}$ the unit vector defining the $z$-coordinated axis. The in-plane velocity component at the upper surface $u\left(x, z=H_{t} / 2\right)$ vanishes, i.e. $u\left(x, z=H_{t} / 2\right)=0$.

On the bottom boundary $z=-H_{t} / 2$, the velocity vanishes, i.e. $\mathbf{v}\left(x, z=-H_{t} / 2\right)=\mathbf{0}$, whereas in both free boundaries $x=L_{t} / 2$ and $x=-L_{t} / 2$ the traction is assumed to vanish, i.e. $\boldsymbol{\sigma} \cdot \mathbf{e}_{x}=\mathbf{0}$. The Stokes equations for an incompressible Newtonian fluid read:

$\nabla p=\eta \cdot \Delta \mathbf{v}$

where $\mathbf{v}$ is the velocity vector with components $\mathbf{v}=(u, w)$ and $p$ the pressure field.

Using the lubrication hypotheses described in Appendix A, the pressure field results

$p(x)=\frac{6 \eta \mathcal{W}}{H_{t}^{3}}\left(\frac{L_{t}^{2}}{4}-x^{2}\right)$
Fig. 6 Real prepreg surface measured with the profilometer (left) and example of extracted profile (right) where only the relative height $\mathrm{z}(\mathrm{m})$ applies (and not the absolute one)
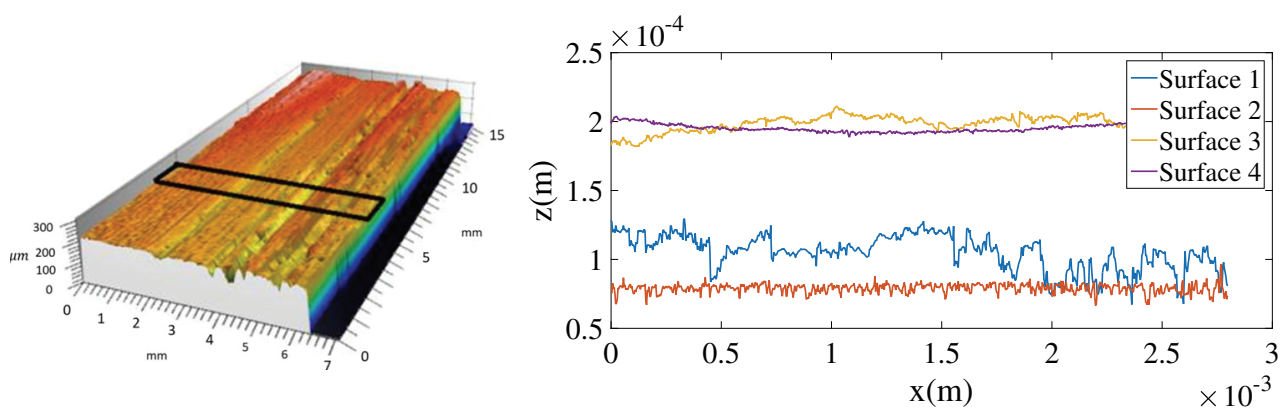
Fig. 7 Laser input model (left) and Roller pressure Field corresponding to a $600 \mathrm{~N}$ force (dimensions: $3 \mathrm{~cm} \times 1.5 \mathrm{~cm}$ Value of Max Pressure : 5MPa) (right)
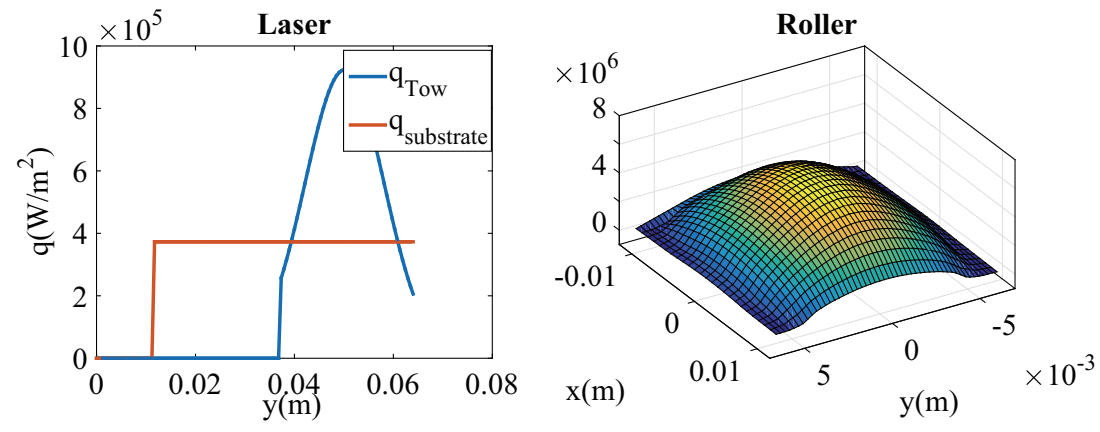

and the compression rate comes from the applied load according to

$\mathcal{W}=\frac{\mathcal{F} H_{t}^{3}}{A L_{t}^{3} \eta}$

Thus, the integration procedure consists of:

- Giving the initial length and thickness $L_{0}$ and $H_{0}$ respectively, or with both having been calculated at time $t, L_{t}$ and $H_{t}$;

- Knowing the applied load $\mathcal{F}$, calculating the squeeze rate $\mathcal{W}$ from Eq. 6;

- Updating the thickness at time $t+\Delta t$ from

$H_{t+\Delta t}=H_{t}-\mathcal{W} \Delta t$

- Updating the rectangle length at time $t+\Delta t, L_{t+\Delta t}$, considering the mass conservation

$L_{t+\Delta t}=\frac{L_{t} H_{t}}{H_{t+\Delta t}}$

- Calculating the gradient of the pressure field Eq. 5 at each coordinate $x \in\left[-L_{t+\Delta t} / 2, L_{t+\Delta t} / 2\right]$, and from it the local velocity field $u(x, z)$ from Eq. 20 , as well as its partial derivatives $\frac{\partial u}{\partial x}$ and $\frac{\partial u}{\partial z}$ that constitute the first row of the gradient of velocity tensor $\nabla \mathbf{v}$. Its second row is composed of $\frac{\partial w}{\partial x}=0$ and $\frac{\partial w}{\partial z}=-\frac{\partial u}{\partial x}$, the latter ensuring the flow incompressibility;

- Coming back to the second item above.

\section{Power-law fluid}

When considering a power-law constitutive equation, the viscosity depends on the equivalent strain rate

$D_{e q}=\sqrt{2(\mathbf{D}: \mathbf{D})}$,

with $\mathbf{D}$ the rate of strain tensor, from

$\tau=\mu \cdot D_{e q}^{n-1} \cdot \mathbf{D}$

where $\mu$ and $n$ are material parameters.

By using the lubrication hypotheses described in Appendix B the integration procedure consist of:

- Given the initial length and thickness of the fluid column: $L_{0}$ and $H_{0}$ respectively, or with both having been calculated at time $t, L_{t}$ and $H_{t}$.

- Knowing the applied load $\mathcal{F}$, calculate the squeeze rate $\mathcal{W}$

Table 1 Value of different simulation parameters, with $C p$ the specific thermal capacity; n the power index [19]; $K_{a}$ and $K_{m}$, respectively the air and composite (in the plane perpendicular to fibers direction) thermal conductivity; $h_{\text {air }}, h_{\text {mould }}$ and $h_{\text {roller }}$ the thermal exchange coefficients between the composite and respectively the air, mould and roller; $\mathcal{F}$ the applied force; $V_{\text {laser }}$ the laying head velocity; $P_{\text {laser }}$ the laser power; $m$ the number of plies; and $T_{a m b}$ and $T_{\text {mould }}$ the temperature of the air and the mould respectively

\section{Parameters of simulation}

\begin{tabular}{llll}
\hline$\rho C p$ & $2.210^{6}$ & $\mathcal{F}$ & $600 \mathrm{~N}$ \\
$n$ & 0.65 & $K_{m}$ & $0.5 \mathrm{~W} /(\mathrm{m} \mathrm{K})$ \\
$K_{a}$ & $0.024 \mathrm{~W} /(\mathrm{mK})$ & $h_{c}$ & $4000 \mathrm{Km} / \mathrm{W}$ \\
$h_{\text {air }}$ & $10 \mathrm{~K} \mathrm{~m}^{2} / \mathrm{W}$ & $h_{\text {mould }}$ & $2500 \mathrm{Km} \mathrm{m}^{2} / \mathrm{W}$ \\
$h_{\text {roller }}$ & $2000 \mathrm{~K} \mathrm{~m}^{2} / \mathrm{W}$ & $V_{\text {laser }}$ & $0.1 \mathrm{~m} / \mathrm{s}$ \\
$m$ & 6 & $T_{\text {amb }}$ & $25 \mathrm{C}$ \\
$T_{\text {Mould }}$ & $25 \mathrm{C}$ & $P_{\text {laser }}$ & $720 \mathrm{~W}$ \\
\hline
\end{tabular}


- Update the thickness at time $t+\Delta t$ from

$$
H_{t+\Delta t}=H_{t}-\frac{\mathcal{W}}{2} \Delta t
$$

- Update the rectangle length at time $t+\Delta t, L_{t+\Delta t}$, invoking the mass conservation

$$
L_{t+\Delta t}=\frac{L_{t} H_{t}}{H_{t+\Delta t}}
$$

- Calculate the gradient of the pressure field at each coordinate $x \in \Omega_{x}(t)$, and from it the local velocity field $u(x, z)$ from Eq. 27, as well its partial derivatives $\frac{\partial u}{\partial x}$ and $\frac{\partial u}{\partial z}$ that constitutes the first row of the gradient of velocity tensor $\nabla \mathbf{v}$. Its second row is composed by $\frac{\partial w}{\partial x}=0$ and $\frac{\partial w}{\partial z}=-\frac{\partial u}{\partial x}$, the last ensuring the flow incompressibility. The velocity gradient allows computing the equivalent strain rate;

- Come back to the second item above.

\section{Thermal analysis of the evolving surface}

As previously indicated, the evolution of the geometry initially described by using the Haar wavelets is carried out by squeezing sequentially all the rectangular elements, as soon as they enter in contact with the upper plate moving down and then compressing them. The solution procedure is quite simple. We consider all the rectangles in contact with the compression plate and solve in these rectangles the squeeze flow model just described for a single rectangular element by assuming that the pressure in all the elements that are not in contact with the compression plate vanishes. As soon as the pressures are available in all the rectangles that are being compressed, the velocity field and more precisely the flow rates can be obtained at the lateral boundaries. The fluid leaving each rectangular element that is being compressed is transferred to the neighbour rectangular element that increases its height accordingly in order to ensure the mass conservation.

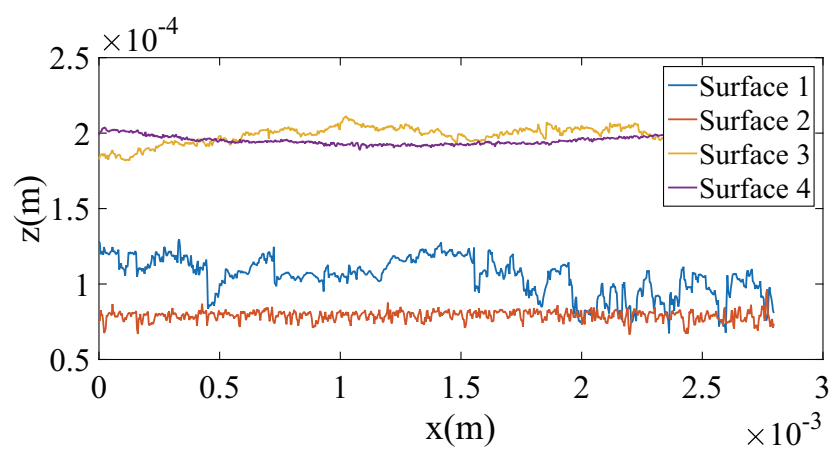

Fig. 8 Real prepreg surfaces
If a compression rate is enforced the process continues until removing all the air content, independently of the force to be imposed to ensure the compression. However, if the compression occurs by applying a given compression force, the compression progresses while the applied forces allows squeezing the fluid. If a thermal coupling is present, as soon as the temperature decreases too much, the viscosity increases accordingly and the squeeze flow almost stops, then the compression rate becomes negligible. Thus, if compression only applies for a short period of time, a residual porosity is expected to persist at the end of the process.

Obviously during the solution process the thermal problem and the squeeze flow one are solved at each time step. The solution of the former allows computing the temperature-dependent viscosity, that is an input in the squeeze flow model. Then, as soon as the surface is updated, the associated thermal conductivity must be expressed in a separated form according to Eq. 2 where the number of terms required in the approximation $n_{r}$ can evolve during the compression.

It is important to note that when considering a null pressure in the rectangles that are not being compressed and because the 2D approximation here addressed, the flow going from a rectangular element towards its two neighbours is no more than the fluid volume displaced by the compression and then the model presented in the previous section seems too rich for the real modelling purposes. However, as soon as the compression is driven by a pressure and not by a compression rate the accurate calculation of the pressure field is compulsory. Moreover, as the pressure depends on the fluid rheology, and at its turn the viscosity depends on the velocity gradient, finally in the general case of a power-law fluid and a compression driven by a given applied load, the previously presented modelling takes all its sense.

\section{ATP process modelling}

In order to understand and take advantage of the effect of the different material and process parameters on the

Table 2 Surfaces roughness parameters according to ISO 4287

\begin{tabular}{llll}
\hline Surface & $R_{a}$ & $R_{t}$ & $R_{s}$ \\
\hline & $\left(10^{-6} m\right)$ & $\left(10^{-6} m\right)$ & $\left(10^{-6} m\right)$ \\
S1 & 2.7 & 34.0 & 43 \\
S2 & 2.1 & 25.3 & 13 \\
S3 & 0.8 & 10.5 & 103 \\
S4 & 0.4 & 4.5 & 57 \\
\hline
\end{tabular}

Note : these values correspond to the micro-roughness 
Fig. 9 Influence of the laser power
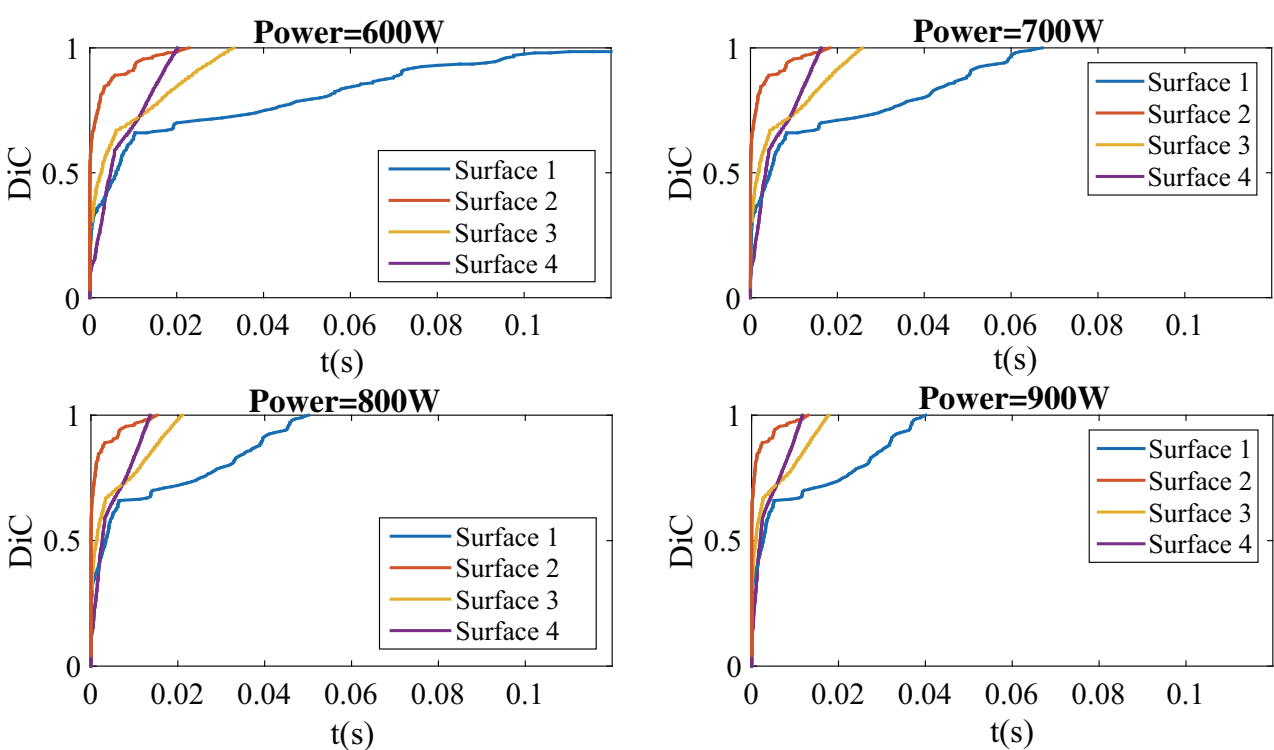

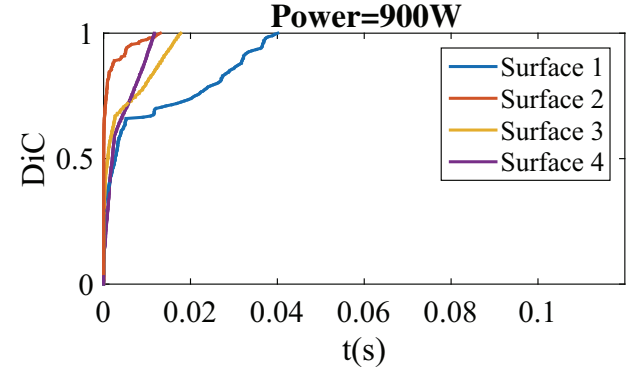

consolidation occurring in the ATP process, we consider the following procedure using the tools previously introduced, and taking into account the following hypotheses:

1. The process is analyzed in a Eulerian framework (the tape and substrate move in the frame attached to the laying head) where the tape cross-section is heated, compressed before cooling, as illustrated in Fig. 5. The domain consists of a laminate composed of $\mathrm{m}$ plies (at least 6 plies in order to minimize the effect of the boundary conditions on the substrate-tool interface);

2. The prepreg surface is measured by using a profilometer, and it is represented as a succession of $1 \mathrm{D}$ profiles (see Fig. 6);
3. The initial temperature of the part is the ambient temperature

4. The laser is considered as a surface thermal source applying on the substrate/incoming tape interface with the flux repartition depicted in Fig. 7

5. The roller applies a pressure on the tape whose space distribution is depicted in Fig. 7. The compaction effort is evaluated to $600 \mathrm{~N}$.

6. The other parameters considered in the simulations are given in Table 1

7. The thermal properties of the prepeg are homogenized and the viscosity obeys a power law, with the thermodependence given by:

$\mu=A \exp B / T$
Fig. 10 Influence of placement head velocity $V_{\text {laser }}$
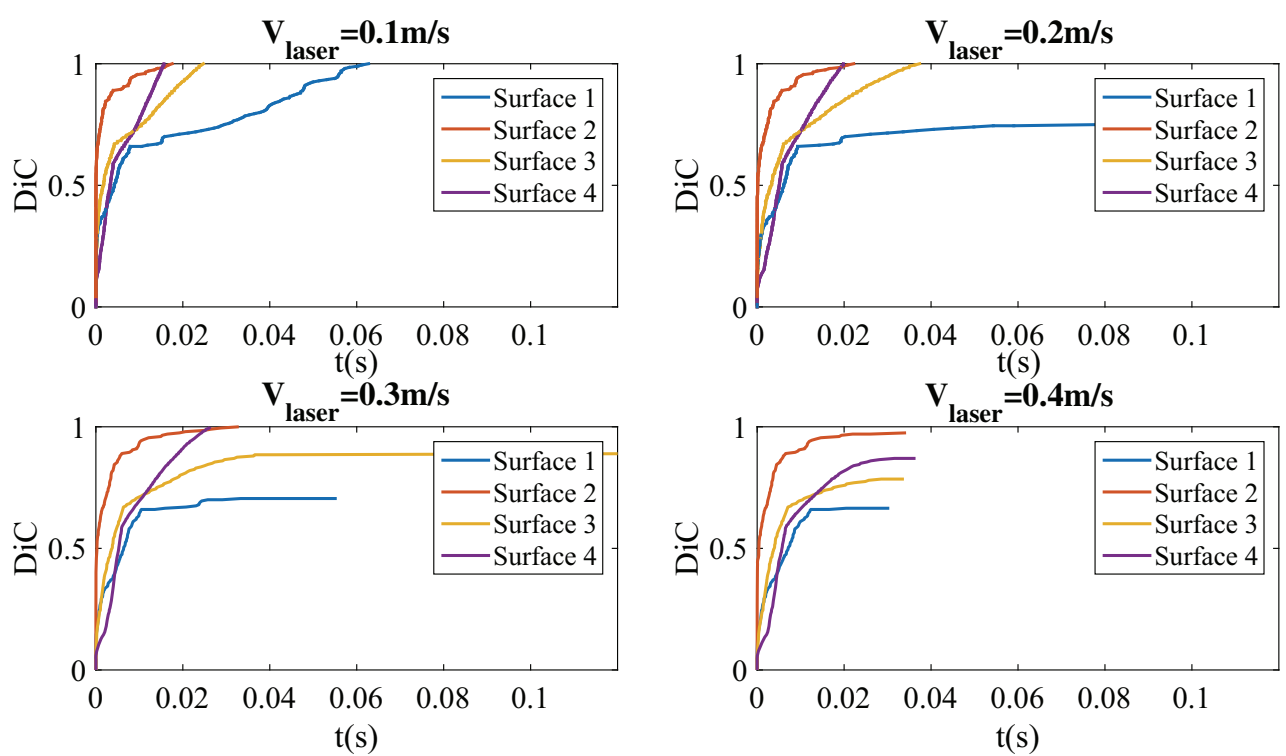
Fig. 11 Influence of the applied force
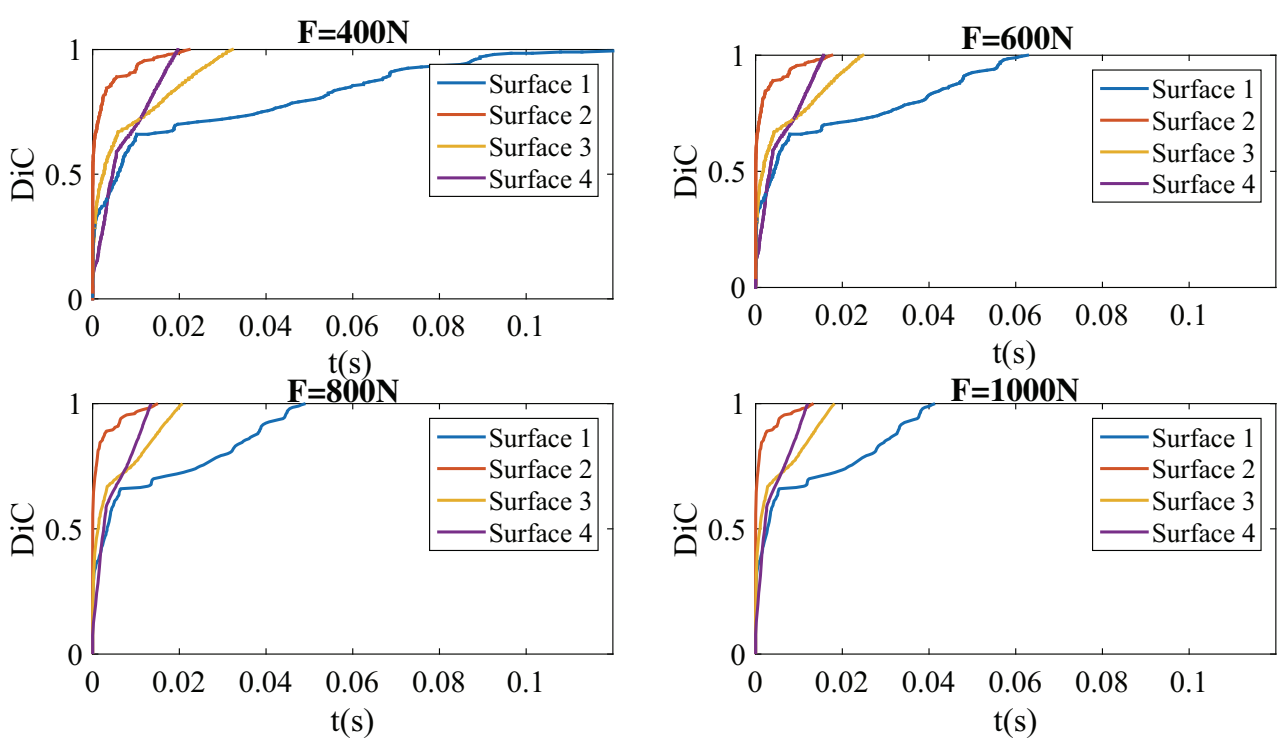

with $A=130$ and $B=2969$, with the viscosity $\mu$ in $P a . s$, and the temperature $T$ in Kelvin [19].

\section{Numerical results: Influence of the material and process parameters on the consolidation evolution}

For the study, four different UD (unidirectional) prepregs from different providers were considered. One profile of each is represented in Fig. 8. The four UD prepreg are made of the same type of material: PEEK matrix (34\%wf) and carbon fibers reinforcement but have been obtained with different processes of impregnation which has a strong influence on their final topographic properties. For better appreciation, the standard roughness properties are indicated in Table 2. These four surfaces have been particularly chosen because one exhibits a very high $R_{t}$ (maximum height of the profile) - profile $\mathrm{S} 1$ - or a very low one - profile S4; more or less important values of $R_{a}$ (arithmetical mean deviation); and different values of $R_{S}$ (mean value of the width of the profile elements). Because the ATP process involves many different parameters, in what follows we perform a sensibility analysis of their effect on the consolidation. The first parameters implied are the process ones: the laser power $P_{\text {laser }}$, the placement head velocity $V_{\text {laser }}$ and the applied load $\mathcal{F}$.
Fig. 12 Influence of the specific thermal capacity $\rho C p$
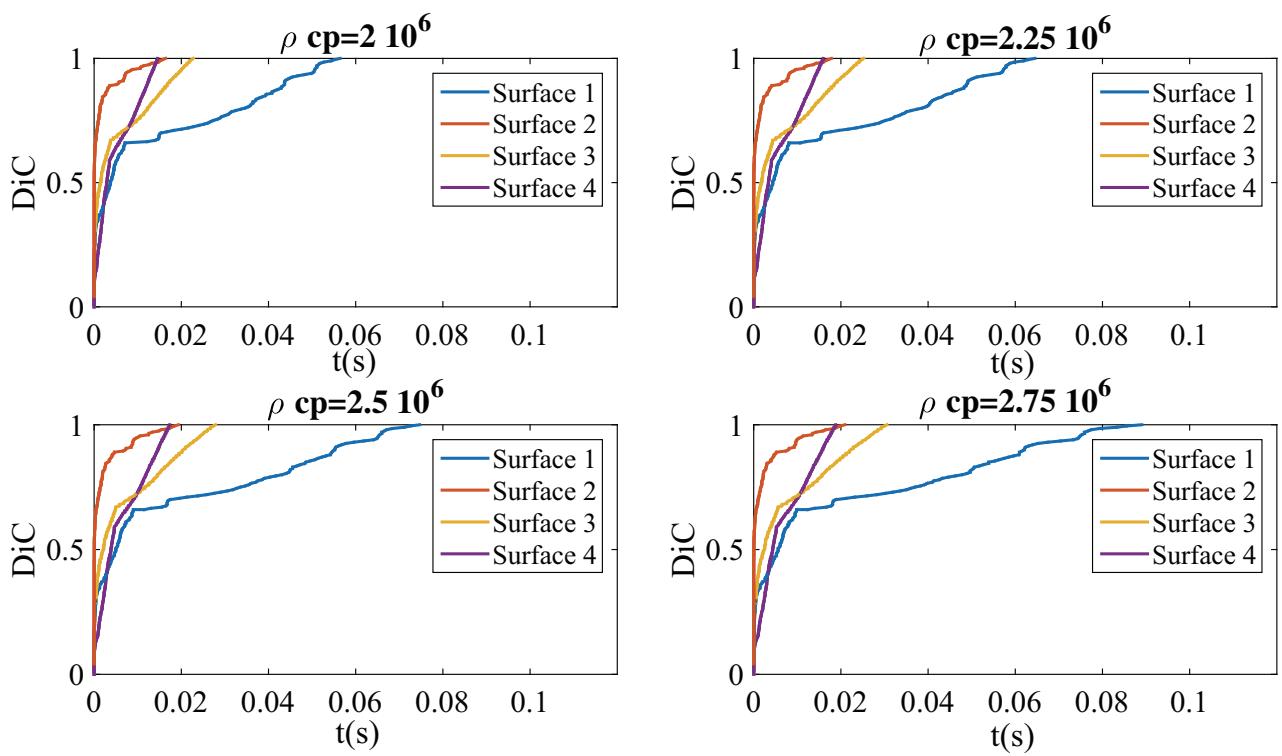
Fig. 13 Influence of thermal conductivity
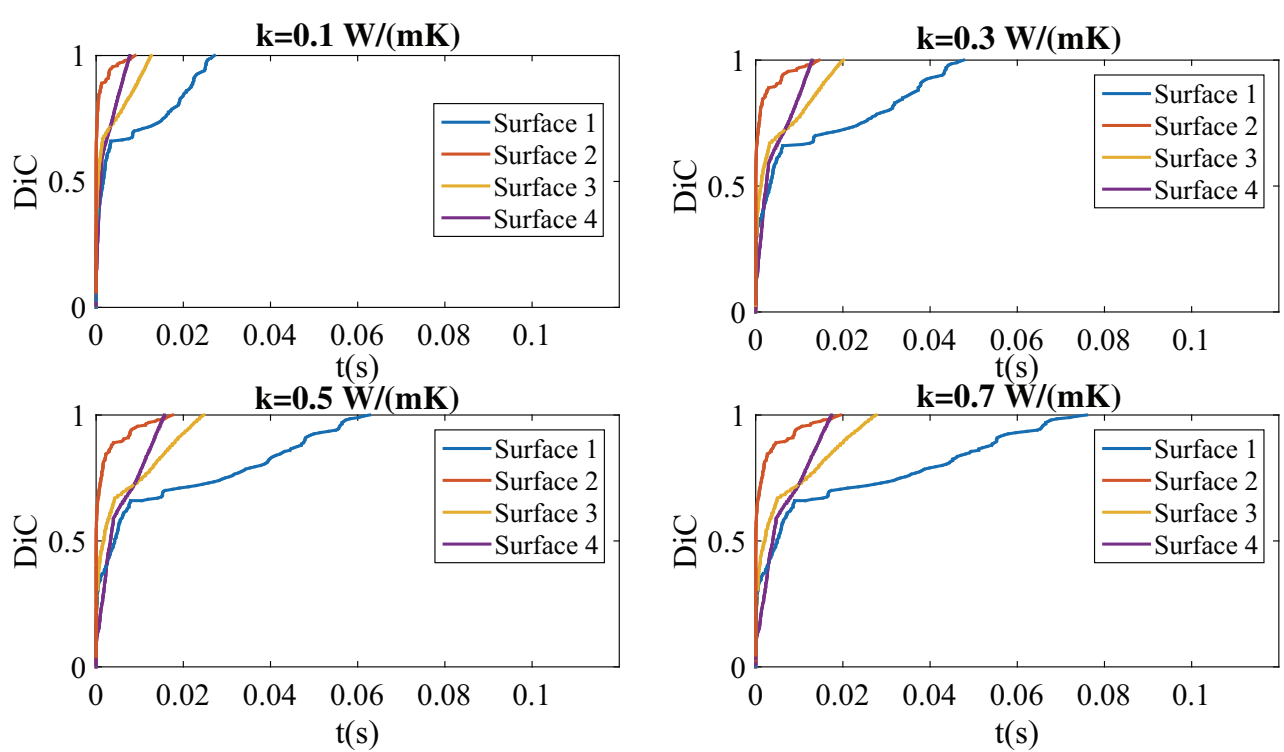

We can see in Figs. 9, 10 and 11, that the perfect consolidation is reached after a time period that depends on the considered surface. In all cases the Surface 1 exhibits the longest time period to reach a perfect consolidation, whereas Surfaces 2 and 4 show the shortest time in all cases. As expected, for a fixed set of parameters, the higher the laser power, the faster the consolidation is attained. In the same way the lower the placement head velocity is, the faster consolidation is reached. Considering the applied force by the compaction roller, the higher the force, the better the consolidation occurs. Nonetheless, it is important to remark that the polymer degradation is not considered in the present work (but could easily be computed) that only pretends to identify the more relevant parameters for a deeper study.
Another remark concerning Figs. 9, 10 and 8, is that the time for reaching $D I C=1$ is more or less affected by the variation of the parameters, in particular surface $\mathrm{S} 1$ is always very affected by the parameters evolution, whereas the other three $S 2, S 3$ and $S 4$ seem notably less influenced.

Then we proceed to modify thermal properties: the specific thermal capacity $\rho C p$ and $K_{m}$, the homogenized through-the-thickness composite thermal conductivity. Figures 12 and 13 also prove that surface $\mathrm{S} 1$ is quite sensitive to the parameters change whereas the other three (S2, S3 and S4) seem much less sensitive.

Thus, increasing $\rho C p$ and the thermal conductivity do not improve consolidation. In fact, lower conductivities allow keeping the interface very hot for a long period with a favourable impact in the squeezing flow, because

Fig. 14 Influence of viscosity $\mu$
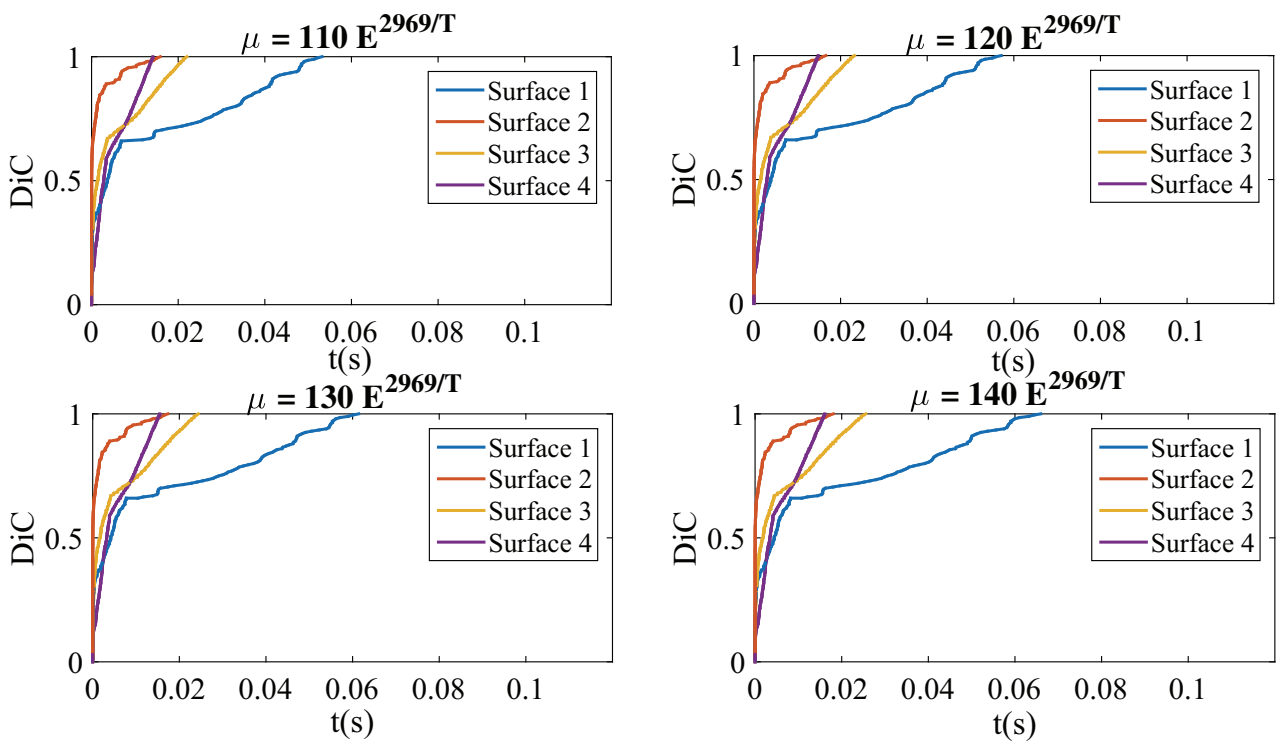

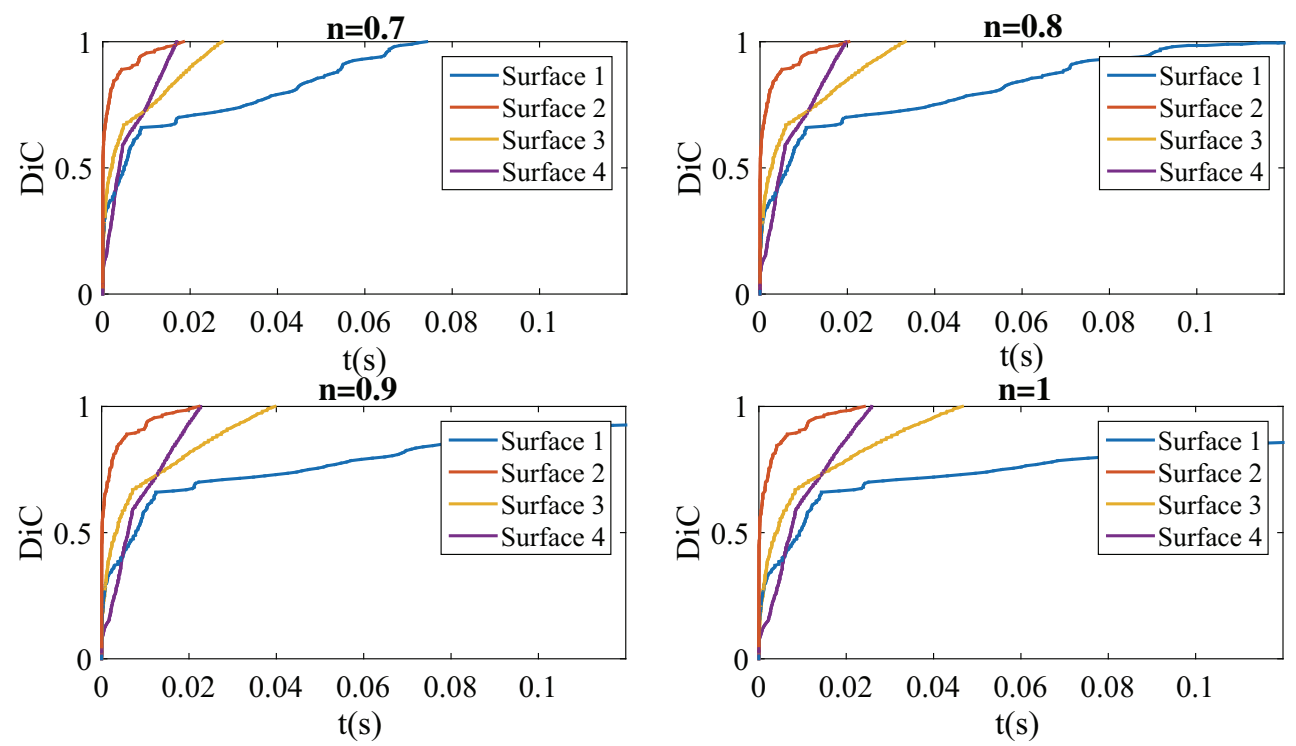

Fig. 15 Influence of power-index $n$

of the lower viscosity. By increasing the conductivity the heat is transferred to the air or the tool and the viscosity at the interface level becomes very fast too high for ensuring the interface compression. Figures 14 and 15 depict the evolution of consolidation with the evolution of the viscosity parameters. As $\mu$ increases consolidation becomes difficult because the applied pressure is not enough for ensuring the adequate flow. On the other hand, by decreasing the power law index $n$, the consolidation time decreases, proving the advantage of using power-law nonNewtonian fluids.

Finally, Fig. 16 proves that when the substrate is composed of few plies, the tool impact is quite relevant, and the fast cooling that it induces has a negative consequence on the consolidation.
In order to better conclude on the effect of the different parameters we define a dimensionless coefficient $I$ that will serve to evaluate the influence of each parameter

$I=\frac{\int_{0}^{t_{r e f}} D I C(t) d t}{t_{r e f}}$

where $t_{\text {ref }}$ (here $t_{\text {ref }}=0.1$ ), represents the time at which both surfaces reach an almost perfect consolidation, and the sensivity index $\mathcal{S}$

$\mathcal{S}(\%)=\frac{I^{\operatorname{Ini+\Delta }}-I^{\operatorname{Ini}}}{\mathcal{P}^{\operatorname{In} i+\Delta}-\mathcal{P}^{\operatorname{Ini} i}} \frac{\operatorname{In}^{\operatorname{Ini}}}{I^{\operatorname{ma}}} \times 100$

where $\mathcal{P}$ is a given parameter with respect to which the sensitivity analysis is performed, $\bullet^{I n i}$ indicates the initial
Fig. 16 Influence of number of the plies
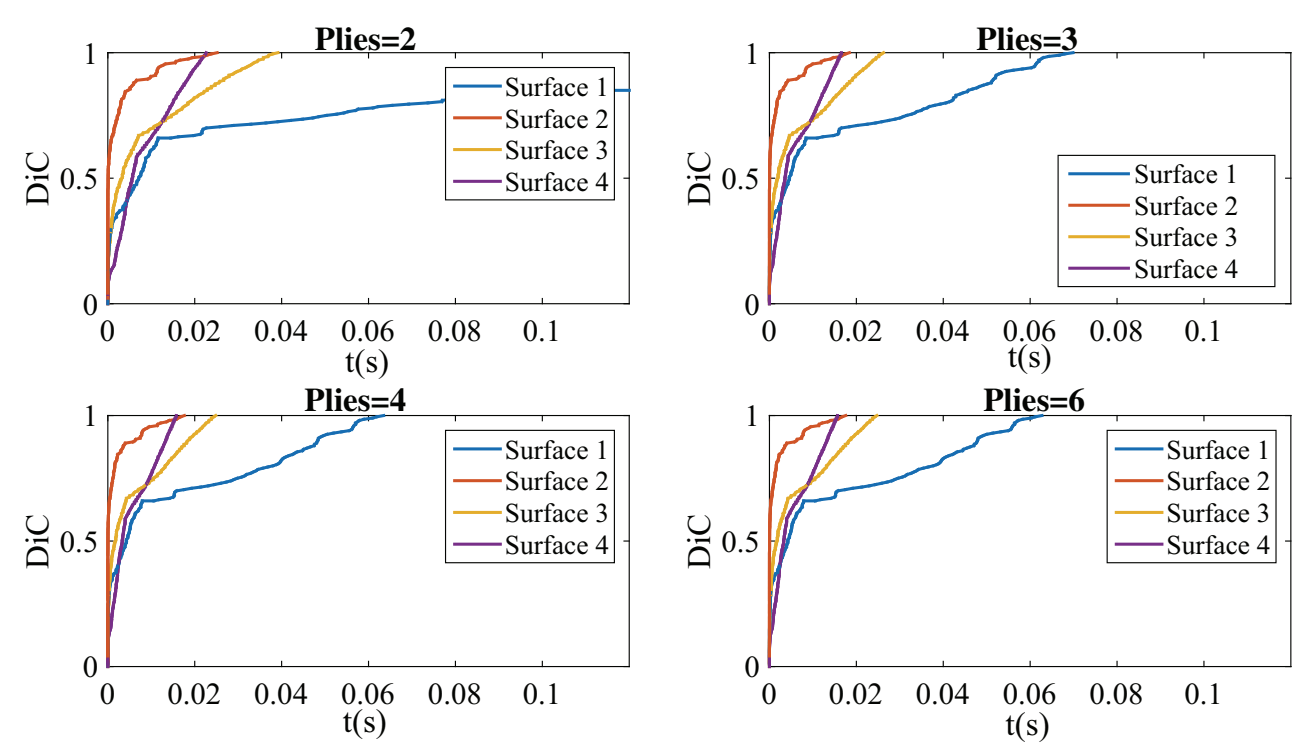


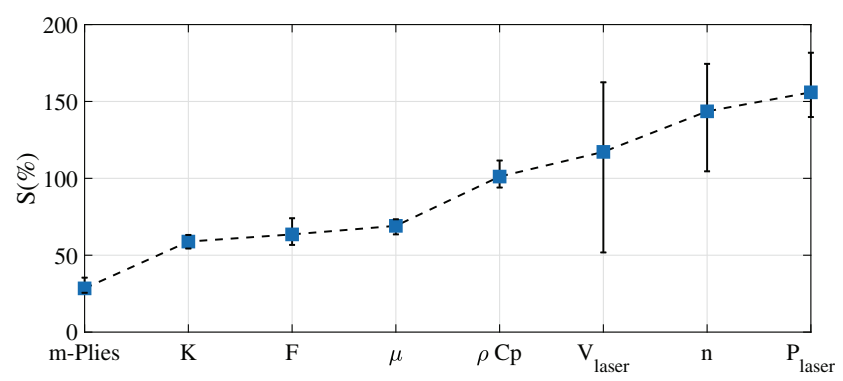

Fig. 17 Sensitivity of each material or process parameters on the evolution of the DIC

state and $\bullet^{\operatorname{Ini}+\Delta}$ a change $\Delta$ of its initial value. Table 3 collects the different sensitivity index for the different surfaces and parameters.

Table 3 highlights the important effect of the laser power, the non-newtonian comportement of the resin and the velocity of deposition on consolidation. These three parameters are the most critical regarding their impact on the consolidation evolution, and moreover they exhibit a large variability depending on the initial surface topography. Figure 17 adds the variability of the four surfaces regarding a variation in a process or material parameter $( \pm 25 \%$ applied on reference parameters of Table 1$)$. In comparison the other parameters evolve quite homogeneously regarding the initial surface properties, and have a more moderated impact on the consolidation evolution, even if it is not negligible at all as represented in the Fig. 17.

We can also conclude that the smoother the surface is (see Fig. 6) the less the consolidation is impacted by the parameters change: Surface 3 and 4 exhibit exactly the same behaviour regarding the sensitivity $\mathcal{S}$ defined above, and Surface 2 always needs the lowest quantity of energy to attain consolidation (see Fig. 18). On the contrary Surface 1 always need the more important quantity of energy to be squeezed and to attain perfect intimate contact.

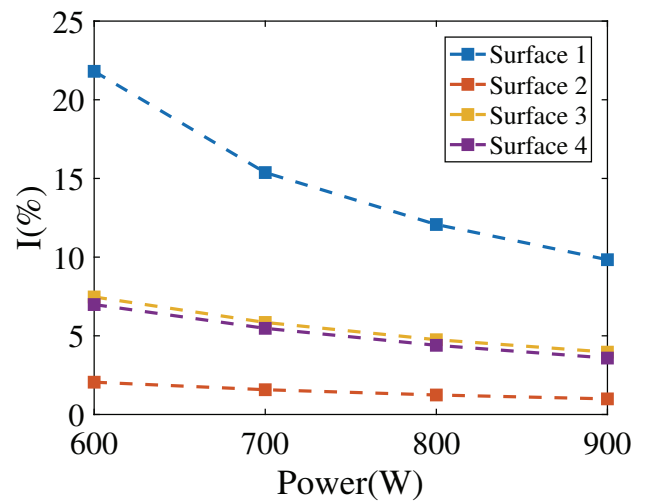

Fig. 18 Evolution of index $I(\%)$ with respect to $P_{\text {laser }}$ and $\mathcal{K}$
Table 3 Impact of $25 \%$ of parameter change on index $\mathcal{S}(\%)$

\begin{tabular}{lllll}
\hline Parameters $\mathcal{P}$ & Surface 1 & Surface 2 & Surface 3 & Surface 4 \\
\hline$m:$ number of plies & 35 & 27 & 26 & 25 \\
$K$ & 57 & 63 & 54 & 61 \\
$\mathcal{F}$ & 74 & 64 & 57 & 60 \\
$\mu$ & 73 & 72 & 63 & 67 \\
$\rho C p$ & 111 & 102 & 94 & 96 \\
$V_{\text {laser }}$ & 52 & 115 & 139 & 162 \\
$n$ & 174 & 104 & 149 & 146 \\
$P_{\text {laser }}$ & 181 & 157 & 140 & 145 \\
\hline
\end{tabular}

\section{Conclusions}

In this work, an efficient modelling of ATP processes based on the high-resolution solution of the coupled thermal-squeeze flow problem at the substrate / incoming tape level was proposed and efficiently solved within a separated representation framework. Real surfaces were measured and accurately represented by using a multiscale analysis based on the use of the Haar wavelets. Finally, four pre-impregnated surfaces, exhibiting various roughness properties, were considered and the sensibility of the degree of intimate contact to different materials and process parameters were analysed. Some conclusions were in agreement with the expected behaviour, however, other were less evident, as for example the influence of roughness, the thermal conductivity or the number of plies in the substrate needing for further analysis already in progress.

\section{Compliance with Ethical Standards}

Conflict of interests The authors declare that they have no conflict of interest.

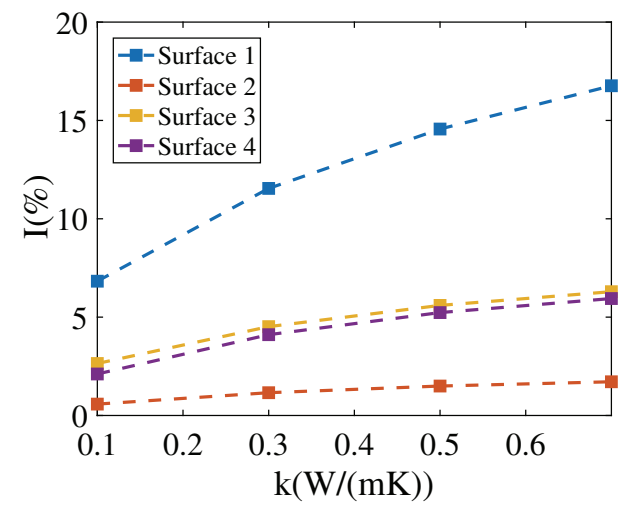




\section{Appendix}

\section{A Squeezing flow of a Newtonian fluid}

Stokes flow model, described by Eq. 4, results in the following scalar equations

$$
\left\{\begin{array}{l}
\frac{\partial p}{\partial x}=\eta\left(\frac{\partial^{2} u}{\partial x^{2}}+\frac{\partial^{2} u}{\partial z^{2}}\right) \\
\frac{\partial p}{\partial z}=\eta\left(\frac{\partial^{2} w}{\partial x^{2}}+\frac{\partial^{2} w}{\partial z^{2}}\right)
\end{array} .\right.
$$

When $H_{t} \ll L_{t}$ the following hypotheses (the so-called lubrication hypotheses) apply, i.e.

$\frac{\partial u}{\partial z} \gg \frac{\partial u}{\partial x}$,

and the velocity components in the thickness direction is almost negligible, i.e. $w \approx 0$, from which the Stokes Eq. 16 are reduced

$\left\{\begin{array}{l}\frac{\partial p}{\partial x}=\eta \frac{\partial^{2} u}{\partial z^{2}} \\ \frac{\partial p}{\partial z}=0\end{array}\right.$.

Now, by integrating the first Eq. 18 with respect to $z$ and taking into account that according to the second relation in Eq. $18 p=p(x)$, as well as the non-slip boundary conditions

$\left\{\begin{array}{l}u\left(x, z=H_{t} / 2\right)=0 \\ u\left(x, z=-H_{t} / 2\right)=0\end{array}\right.$,

the result is

$u=\frac{1}{2 \eta} \frac{d p}{d x}\left(z^{2}-\frac{H_{t}^{2}}{4}\right)$

whose integration in the thickness allows for the calculation of the flow rate $q$

$q=\int_{-H_{t} / 2}^{H_{t} / 2} u d z=-\frac{1}{12 \eta} \frac{d p}{d x} H_{t}^{3}$

The mass conservation, taking into account the fluid incompressibility and the compression velocity $\mathcal{W}, w(x, z=$ $\left.H_{t} / 2\right)=-\mathcal{W}$, reads

$\mathcal{W}=\frac{\partial q}{\partial x}$,

that allows for deriving the equation related to the pressure field

$\mathcal{W}=-\frac{H_{t}^{3}}{12 \eta} \frac{d^{2} p}{d x^{2}}$
By integrating Eq. 23 and taking into account that pressure vanishes at $x= \pm L_{t} / 2$, the result is

$p(x)=\frac{6 \eta \mathcal{W}}{H_{t}^{3}}\left(\frac{L_{t}^{2}}{4}-x^{2}\right)$

The applied load $\mathcal{F}$ related to the compression rate $\mathcal{W}$ of the surface of width $A$ can be obtained by integrating the pressure on the upper boundary

$\mathcal{F}=A \int_{-L_{t} / 2}^{L_{t} / 2} p(x) d x=\frac{A L_{t}^{3} \eta \mathcal{W}}{H_{t}^{3}}$,

from which the compression rate can be obtained from the applied load

$\mathcal{W}=\frac{\mathcal{F} H_{t}^{3}}{A L_{t}^{3} \eta}$

\section{B Squeezing flow of a Newtonian fluid}

By considering the Stokes flow model

$\left\{\begin{array}{l}\frac{\partial p}{\partial x}=\mu \frac{\partial}{\partial z}\left(\left(\frac{\partial u}{\partial z}\right)^{n-1} \frac{\partial u}{\partial z}\right) \\ \frac{\partial p}{\partial z}=0\end{array}\right.$,

and by employing the same rationale that in the Newtonian case, the velocity reads

$u=\frac{\partial p}{\partial x} \frac{1}{\mu} \alpha^{(1-n) / n} \frac{n}{n+1}\left(|-z|^{(1+n) / n}-\left(H_{t}\right)^{(1+n) / n}\right)$,

where $\alpha=\frac{1}{\mu} \sqrt{\left(\frac{\partial p}{\partial x}\right)^{2}}$.

Again, mass conservation leads to

$-\frac{2 H_{t}^{2+\frac{1}{n}}}{\mu^{\frac{1}{n}}(1+2 n)}\left(\frac{\partial p}{\partial x}\right)^{-1+\frac{1}{n}} \frac{\partial^{2} p}{\partial^{2} x}=\mathcal{W}$

This equation can be discretized by using any state of the art numerical technique, like finite elements, finite differences, ... with the support of a grid composed of $N$ nodes, in which the pressure is stated at each time step. Thus the discrete problem can be expressed in the matrix form:

$\mathbf{K P}=\mathbf{F} \mathcal{W}$

where $\mathbf{K}$ is the so-called $N \times N$ stiffness matrix, and $\mathbf{P}$ the $N \times 1$ vector that contains the nodal approximation of the pressure field. However, in reality only the compression force is known, and the relation between the applied force and the rate of thickness reduction (compression rate) is only explicit in the Newtonian case. Thus, in the present case, the model must be completed with an additional 
relation, the one that expresses the equality of the integral of the pressure and the applied force, that is:

$\int_{-L_{t} / 2}^{L_{t} / 2} A p(x) d x=\mathcal{F}$

whose discrete form reads

$-\mathbf{F}^{T} \mathbf{P}=\mathcal{F}$

by combining Eqs. 30 and 32 it results the linear system

$\left[\begin{array}{cc}\mathbf{K} & -\mathbf{F} \\ -\mathbf{F}^{T} & 0\end{array}\right]\left[\begin{array}{c}\mathbf{P} \\ \mathcal{W}\end{array}\right]=\left[\begin{array}{l}\mathbf{0} \\ \mathcal{F}\end{array}\right]$

\section{References}

1. Barasinski A, Leygue A, Soccard E et al (2013) Identification of non uniform thermal contact resistance in automated tape placement process. Int J Mater Form 7:479-486

2. Bur N, Joyot P, Ghnatios C, Villon P, Cueto E, Chinesta F (2016) On the use of model order reduction for simulating automated fibre placement processes. Adv Model Simul Eng Sci 3:4

3. Chinesta F, Ammar A, Cueto E (2010) Recent advances and new challenges in the use of the proper generalized decomposition for solving multidimensional models. Arch Comput Meth Eng $17: 327-350$

4. Chinesta F, Ladeveze P, Cueto E (2016) A short review on model order reduction based on proper generalized decomposition. Adv Model Simul Eng Sci 18:395-404

5. Chinesta F, Keunings R, Leygue A (2014), The Proper Generalized Decomposition for Advanced Numerical Simulations. SpringerBriefs in Applied Sciences and Technology

6. Chinesta F, Leygue A, Bognet B, Ghnatios C, Poulhaon F, Bordeu F, Barasinski A, Poitou A, Chatel S, Maison-Le-Poec S (2014) First steps towards an advanced simulation of composites manufacturing by automated tape placement. Int J Mater Form $7: 81-92$
7. Daubechies I (1988) Orthonormal bases of compactly supported wavelets. Commun Pure Appl Math 41:909-996

8. Lee W, Springer GS (1987) A model of the manufacturing process of thermoplastic matrix composites. J Compos Mater 21:1057-1082

9. Leon A, Barasinski A, Nadal E, Chinesta F (2015) Highresolution thermal analysis at thermoplastic pre-impregnated composite interfaces. Composite Interfaces 22:767-777

10. Leon A, Perez M, Barasinski A, Abisset-Chavanne E, Chinesta F (2017) On the properties evolution of engineered surfaces of thin reinforced thermoplastic tapes during consolidation. Surf Topogr Metrol Prop 5:044003

11. Leon A, Barasinski A, Chinesta F (2017) Microstructural analysis of pre-impreganted tapes consolidation. Int J Mater Form 10:369-378

12. Levy A, Heider D, Tierney J, Gillespie J (2014) Inter-layer thermal contact resistance evolution with the degree of intimate contact in the processing of thermoplastic composite laminates. J Compos Mater 48:491-503

13. Longuet-Higgins M (1957) Statistical properties of an isotropic random surface. Series A-Math Phys Sci 250:157-174

14. Longuet-Higgins $M$ (1957) The statistical analysis of a random, moving surface. Series A-Math Phys Sci 249:321387

15. Mandelbrot B (1983) The fractal geometry of Nature W.H. Freeman and Company, New York

16. Mandelbrot B, Van Ness J (1968) Fractional Brownian motions, fractional noises and applications. SIAM Rev 10:422-437

17. Mandelbrot B, Passoja D, Paullay A (1984) Fractal character of fracture surfaces of metals. Nature 308:721-722

18. Mandelbrot B (2002) Gaussian self-affinity and fractals. Springer-Verlag, New York

19. Mantell S, Springer G (1992) Manufacturing process models for thermoplastic composites. J Compos Mater 16:2348-2377

20. Shuler S, Advani S (1996) Transverse squeeze flow of concentrated aligned fibers in viscous fluids. J Non-Newtonian Fluid Mech 65:47-74

21. Woo I, Springer G (1987) A Model of the Manufacturing Process of Thermoplastic Matrix Composites. J Compos Mater 21:10171055

22. Yang F, Pitchumani R (2001) A fractal Cantor set based description of interlaminar contact evolution during thermoplastic composites processing. J Mater Sci 36:4661-4671 